\title{
Finding the "right" Canadian Neurology Residency Program During the COVID-19 Era
}

\author{
Ario Mirian (1D, Mary E. Jenkins, Christopher J. Watling, Shannon L. Venance, \\ Anita Florendo-Cumbermack
}

Keywords: Postgrad Medical Education, Neurology - Education, Residency Training

doi:10.1017/cjn.2020.218

Can J Neurol Sci. 2021; 48: 47-49

\section{INTRODUCTION}

The coronavirus disease 2019 (COVID-19) pandemic has disrupted life worldwide, and neurology residency programs and clerkship training are no exceptions. Many institutions have been able to rapidly respond to the ever-changing care needs and delivery models required for effective and safe patient care while continuing to provide neurologic training in a safe and effective manner. ${ }^{1}$ Pandemic-related clerkship disruptions mean medical students face new challenges in navigating the residency selection process.

In this article, we describe barriers and opportunities created by the COVID-19 pandemic for applicants in ranking potential Canadian neurology residency programs. Our purpose is to provide actionable advice for medical students entering upcoming application cycle(s) for adult or pediatric neurology residency programs amidst the unforeseen changes due to COVID-19 (summarized in Table 1). This article may also be of interest to Canadian neurology residency programs who wish to be proactive in preparing for the upcoming application process. We use our collective background in medical education and residency training, at Western University Schulich School of Medicine and Dentistry, to provide recommendations on how applicants may obtain information that will help them to choose the "right" neurology residency program. The examples we offer are not exhaustive but highlight some of the ways that programs and learners might adapt to current challenges.

In any application cycle, applicants are encouraged to evaluate a number of specific factors when selecting or ranking a neurology residency program. Some of these considerations are program specific and remain "fixed" as they are not influenced by the COVID-19 pandemic. This broadly includes but is not limited to the availability of subspecialty and generalist expertise, population catchment area, physical location, and facultyto-trainee ratio. In our experience, the breadth of neurological clinical experiences is enhanced by diverse subspecialty representation among faculty balanced with a generalist approach to the most common neurologic presentations, high patient volumes, and low faculty-trainee ratios. Yet, a good neurology training program need not feature all these characteristics.
A broad training experience in clinical neurology still remains fundamental to acquiring the knowledge and clinical skills necessary for independent practice as a neurologist. Other factors are unique to the applicant and influenced by personal preferences such as proximity to a partner and/or family, size of the city, and personal finances and the city's cost of living, to name a few. The article will not expand on these further but will focus on how the applicant can navigate the application cycle during the COVID-19 pandemic.

\section{COVID-Related Factors}

All applicants are encouraged to try to collect relevant data on the academic environment, resident program culture, and their areas of interest to inform their decisions. In usual times this information would be gathered from clinical electives, current or past residents, program directors, faculty and program websites, or personal tours. These components of the residency application process have been altered by the COVID-19 pandemic.

\section{Academic Environment}

The academic environment generally consists of an institution's clinical settings and the culture of the learning and working environment. The immediate impact to an applicant's informed decision-making will be the loss of visiting neurology electives. Electives are a major source of rich data on a program. Unfortunately, visiting electives have been cancelled for the 2020-2021 academic year as per the Association of Faculty of Medicine of Canada (AFMC). ${ }^{2}$ This is due to a variety of reasons including patient and student safety, travel restrictions, the need for quarantining at both ends of the elective, and alterations in patient volumes. The loss of external electives will also mean

From the Clinical Neurological Sciences, Western University, London, ON, Canada (AM, MEJ, SLV, AFC); and Oncology and Clinical Neurological Sciences, Western University, London, ON, Canada (CJW)

Received August 9, 2020. Final Revisions Submitted September 27, 2020. Date of Acceptance September 29, 2020.

Correspondence to: Ario Mirian, Clinical Neurological Sciences, Western University, 339 Windermere Rd., London, ON, N6A 5A5, Canada. Email: amirian2@uwo.ca 


\section{Table 1: Main recommendations for prospective neurology residency applicants}

\begin{tabular}{|c|c|}
\hline \multirow[t]{2}{*}{ Academic Environment } & $\begin{array}{l}\text { - Attend or review virtual information sessions or } \\
\text { recordings of academic half-days }\end{array}$ \\
\hline & $\begin{array}{l}\text { - Ask current residents about the transition to virtual } \\
\text { learning and patient care and how the program has } \\
\text { been successful in meeting educational needs }\end{array}$ \\
\hline \multirow[t]{2}{*}{ Residency Program Culture } & $\begin{array}{l}\text { - Assess ways in which the program has increased } \\
\text { supports for resident wellness and dealt with } \\
\text { unforeseen circumstances due to the COVID-19 } \\
\text { pandemic }\end{array}$ \\
\hline & $\begin{array}{l}\text { - Enquire about a program's mentorship culture as it } \\
\text { is an important source for resident support and } \\
\text { academic guidance }\end{array}$ \\
\hline \multirow[t]{2}{*}{ Areas of Interest } & $\begin{array}{l}\text { - Avoid making the mistake of assessing residency } \\
\text { programs based on the institution's overall } \\
\text { research capacity or prestige }\end{array}$ \\
\hline & $\begin{array}{l}\text { - Ask residency programs how they have supported } \\
\text { current and past residents' career goals and which } \\
\text { areas of clinical exposure are available during the } \\
\text { residency program }\end{array}$ \\
\hline \multirow[t]{2}{*}{ The Unknowns } & $\begin{array}{l}\text { - Find out how residency program selection } \\
\text { committees will address the inequality of } \\
\text { prospective applicants only obtaining letters } \\
\text { of references from their home institution }\end{array}$ \\
\hline & $\begin{array}{l}\text { - Enquire about the dynamic institutional policies } \\
\text { for trainee safety as the COVID-19 case burden } \\
\text { changes geographically across Canada }\end{array}$ \\
\hline
\end{tabular}

that applicants will be only able to obtain reference letters from their home institution. Applicants are encouraged to ask residency programs how their selection committees will address this inequality in the selection process.

There are several strategies that applicants can use to obtain valuable information about residency programs that may inform their decision-making. In the absence of in-person electives, applicants can be expected to turn to program websites for information. We recommend that programs enrich the resources available to applicants on such websites. Programs could also offer the opportunity for prospective applicants to join their virtual academic half-days and/or additional educational sessions. Doing so offers insight not only into the quality of the educational program but also into the program culture, including how faculty and residents interact. Applicants may be able to access virtual meetings or recorded interviews with program directors, neurology faculty, residents, or recent graduates and Q\&A website sections for information about the program and academic environment. Residency programs across the country can collaborate in future application cycles to provide informative virtual session(s) to all potential applicants should this be a successful strategy at the individual institutional level. Virtual tours showcasing the breadth of clinical facilities and opportunities at an institution could be made available to applicants. This may replace institutional tours that typically take place as part of the interview process as in person interviews have been cancelled in Canada. ${ }^{3}$ In addition to evaluating the academic curriculum, applicants are encouraged to gauge the adaptability of neurology residency programs in maintaining high quality education and rising to the challenge of meeting new but needed training in areas such as virtual patient care. ${ }^{4}$ It is helpful to ask current residents how the transition to virtual learning and virtual patient care has been handled and whether online sessions have been successful in meeting their educational needs. Programs that will be successful in this educational transition likely have a well-established foundation in clinical and teaching excellence.

\section{Residency Program Culture}

An underappreciated component of residency training is identifying whether the culture of the residency program is supportive and inclusive. Beyond visiting electives, networking at conferences or pre-interview socials is a useful way to get a sense of your fit while experiencing important aspects such as resident culture and camaraderie. We suggest that applicants look for evidence of a program's engagement in nurturing a supportive culture, such as their efforts to connect applicants with existing residents. We encourage applicants to take advantage of any virtual information session offered by a residency program to get a sense of resident experiences and how they view their faculty support. Ask about resident participation in educational sessions including virtual learning and look at the balance of resident and faculty teaching, and the engagement of faculty in collaborating with residents on crafting educational sessions.

An important factor to consider is how programs are supporting resident wellness during the COVID-19 pandemic. We recommend asking how the program, department, and institution have increased supports for resident wellness during this time of uncertainty. Current residents are a helpful source of information in assessing whether the program has had effective and supportive communication with trainees. Equally important, is a program's flexibility in handling unforeseen personal circumstances that have affected trainees such as reduced availability of childcare and dealing with residents who have medical conditions that put them at greater risk if they were to contract COVID. Ask how the program adapted its rotations and clinical structure to deal with the increased workload while ensuring that residents meet their training requirements. Information on a program's mentorship culture should also be sought because it is a source of resident support and academic guidance for trainees which is particularly important during these uncertain times. Applicants might also ask about school-wide wellness supports, such as counseling services and educational accommodations.

\section{Areas of Interest}

According to a recent nationwide survey, $90 \%$ of graduating neurology residents in the USA planned to pursue a fellowship after residency. ${ }^{5}$ Interestingly, only $63 \%$ planned to continue in an academic practice, and $46 \%$ did not feel that they had enough outpatient exposure before they were required to apply for a fellowship. This highlights that even for residents in usual times, it is difficult to decide on a career path. It is not surprising that prospective applicants may find it difficult to foresee how a residency program can meet their career aspirations as these often change beyond medical school. Importantly, it is not an expectation for applicants to have specific career plans at the time of application and/or interview. Instead, we recommend applicants evaluate the culture of mentoring and faculty support provided 
for trainees to attain their individual career goals. Ask trainees how they are/were supported in meeting their professional goals by asking for examples. Those with an interest in exploring general neurology practice should inquire about generalists on faculty, supports for community rotations, and likewise for public health opportunities as it relates to neurological disease for applicants with a global health interest. If an applicant is seeking a research-intensive career, we recommend getting an overview of subspecialty fellowships that past residents have attained. This can provide insight to the program's support system in place to pursue competitive fellowship positions. This should not be mistaken for assessing residency programs solely based on the institution's overall research capacity or prestige. It is not uncommon for many neurology programs, regardless of institutional reputation, to provide trainees with outstanding opportunities for protected research time. These experiences frequently offer appropriate introduction to clinical research while allowing neurology trainees to be competitive for desired fellowships. We recommend those with a strong interest in pursuing highly involved research projects to inquire about research mentorship.

We anticipate that the COVID-19 pandemic will continue to impact neurological research in many domains. Many institutions may pivot research initiatives to address societal needs that will arise from this pandemic while some research projects have been put on hold. Our view is that current and future neurology residents will still be able to adequately explore academic interests appropriate for their level of training in basic science or clinical research while having new opportunities in public health, quality improvement, and education research. Education research is exploding as neurologic education is transforming, thanks to changes from Competency Based Medical Education, neurologic practice, and COVID-19. Change is a great fodder for research questions. We encourage applicants to ask residency programs about the institutional changes in research capacity, how their research interests can be met, and upcoming public health opportunities relating to neurological disease. Directly inquiring about how a program is ensuring strong research training despite institutional disruptions will likely yield useful information.

\section{Addressing the Unknowns}

The fight against the COVID-19 pandemic continues to have unknowns and challenges. With this comes the opportunity for applicants to be proactive about seeking a training program that aims to provide excellent and safe education, training, and patient care despite the uncertainty. Recognize that programs currently at epicenters of COVID-19 will have lessons learned in responding to trainee safety and learning needs; however, the geographical burden of cases will continue to change and impact residency programs at different times. We recommend inquiring about institutional policies that help ensure trainee safety during clinical service. These may include modifications to team composition on inpatient service and consults, acute stroke protocols, off-service, and smaller community rotations, etc. Residents are deeply involved in the pandemic response on multiple levels. Institutions and centers that include resident participation in policy adaptation and decision-making, reflect an excellent training environment for learning and leadership.

\section{Conclusion}

The COVID-19 pandemic will place additional burden on medical students to effectively navigate the residency selection process. Institutions that are proactively trying to create practical solutions will allow potential applicants to have an accurate overview of the residency program and provide novel ways to address questions. An actionable plan that focuses on ensuring that the academic environment and program culture are innovative and trainee centered will help medical students identify the "right" neurology residency program for their individual career and wellness goals.

\section{ACKNOWLEDGMENTS}

None.

\section{Disclosures}

Shannon L. Venance reports personal fees from Sanofi Genzyme, outside the submitted work.

The remaining authors have nothing to disclose.

\section{Statement of Authorship}

AM: Design and conceptualization, literature review, drafted the manuscript

MEJ: Critical revision of the manuscript for intellectual content

SLV: Critical revision of the manuscript for intellectual content

CJW: Critical revision of the manuscript for intellectual content

AFC: Design and conceptualization, drafted the manuscript

\section{REFERENCES}

1. Agarwal S, Sabadia S, Abou-Fayssal N, et al. Training in neurology: Flexibility and adaptability of a neurology training program at the epicenter of COVID-19. Neurology. 2020;94:1-e7

2. Association of Faculties of Medicine of Canada (AAFMC). Visiting Electives for Class of 2021. In: News [online]. Available at: https://service.afmcstudentportal.ca/index.php?/News/NewsItem/ View/163/visiting-electives-for-class-of-2021-stages-a-optionvisiteurs-pour-la-promotion-2021; accessed August 5, 2020.

3. Canadian Residency Matching Service (CaRMS). 2021 R-1 Match Timeline and Interviews. In: 2021 Match Cycle Updates [online]. Available at: https://www.carms.ca/2021-cycle-updates/\#15879 95108804-b181e477-a721; accessed June 24, 2020.

4. Al Gaeed M, Grewal M, Richardson PK, Guerrero CRL. COVID-19: neurology residents' perspective. J Clin Neurosci. Epub 2020 May 4;78:452-453.

5. Mahajan A, Cahill C, Scharf E, et al. Neurology residency training in 2017: a survey of preparation, perspectives, and plans. Neurology. 2019;92(2):76-83. 